\title{
Physical Layer Differential Network Coding for Two-way Relay Channels
}

\author{
Tao Cui \\ FeiFei Gao \\ Chintha Tellambura \\ Department of Electrical Engineering Institute for Infocomm Research Department of Electrical \& Computer Engineering \\ California Institute of Technology \\ Pasadena, CA 91125, USA \\ 1 Fusionopolis Way \\ Email: taocui@caltech.edu \\ \#21-01 Connexis, Singapore 138632 \\ Email: feifeigao@ieee.org \\ University of Alberta \\ Edmonton, Alberta T6G 2V4 \\ Email: chintha@ece.ualberta.ca
}

\begin{abstract}
In this work, we consider differential modulation in two-way relay channels (TWRC). In single antenna systems, we propose non-coherent schemes for both amplify-and forward (AF) and decode-and-forward (DF) where the channel state information is not required. These new schemes are counterparts of the traditional non-coherent detection in point to point communications. The difficulty with differential modulation design in TWRC is that the received signal is a mixture of the signals from both source terminals. We derive maximum likelihood (ML) detectors for both AF and DF. The DF protocol can be considered as performing differential network coding at the physical layer. In addition, we propose several suboptimal alternatives including decision feedback and prediction based detectors. All these strategies work well as evidenced by simulation results. We also extend the schemes to the multiple-antenna case and provide design criterion of differential unitary space time modulation.
\end{abstract}

\section{INTRODUCTION}

Two-way communications is a promising type of modern communications, where both source terminals simultaneously send information to each other. Two-way relay channels (TWRC) has drawn much interest recently [1]-[4]. Both AF and DF under one-way relay channels were extended to the half-duplex TWRC in [1]. In [2], a new type relaying scheme, called partial decode-and-forward was designed for TWRC, and the space-time codes that can achieve full spatial diversity were also proposed. In [3], [4], the relay function is optimized to attain the minimum error probability. Besides, an estimateand-forward scheme is also proposed in [3], [4]. Most work on TWRC [1]-[4] relies on perfect channel state information (CSI), and data recovery uses coherent data detection. In practice, accurate channel estimate may be hard to obtain. In this case, non-coherent schemes, not relying on the instant CSI, is a better choice [5]-[7]. However, differential strategy in TWRC is a new and challenging problem because in TWRC the received signal at one terminal is a mixture of its transmitted signal and the signal from the other terminal. If the self-signal component is known, it can be canceled and the conventional differential scheme [5]-[7] can be applied. However, when both channels are unknown, the mixture of the two unknown parts destroy the phase rotation property which prevents the use of traditional differential transmission.

In this work, we start with a single antenna system. We first consider the AF relaying scheme and derive the probability density function (pdf) of the received signal. Since the pdf cannot be expressed in a simple form, we propose a suboptimal criterion, where the pdf of the received signal conditioned on the unknown signal from the other source terminal can then be described by the modified bessel function giving a maximum likelihood (ML) detector. As the ML detector may be hard to use in practice due to the modified bessel function, we propose a decision feedback scheme by considering three consecutive received signals. Moreover, a prediction based detector is proposed for $\mathrm{AF}$ in time varying channels. In DF, the relay first decodes the signals from the two terminals and re-encodes the information before broadcasting. The so called differential physical layer network coding is proposed, which performs network coding at the physical layer non-coherently. However, different from conventional DF where $s_{1}$ and $s_{2}$ are detected separately at the relay and $s_{i}$ is transmitted signal from source $i, i=1,2$, the proposed strategy directly detects $s_{1}+s_{2}$ and encodes $s_{1}+s_{2}$ directly. In the end, all the proposed schemes are extended to the multiple antennas. The design criterion of DUSTM is also given through performance analysis. From simulation, we find that the proposed protocols are especially useful when the required average data rate is high.

\section{Single Antenna Systems}

\section{A. System Model}

We consider a network with 3 nodes: two source terminals and one relay, where the two source terminals want to exchange information with the help of the relay. We assume a half duplex system. The system is operated in time slots. Each time slot is partitioned into two equal parts. The first part is used for uplink transmission from the sources to the relay and the second part is used for downlink transmission from the relay to the two terminals. We consider a special protocol where the two terminals are active simultaneously in the first part, which corresponds to the 2 time slot protocol in [2]. 4 time slot and 3 time slot protocols are also proposed in [2]. In 4 time slot protocols, each time slot is partitioned into 4 parts. Only one node is active in any part, which actually reduces to the conventional one way communication. Therefore, conventional differential modulations apply to 4 time slot protocols directly. Here, we mainly focus on the 2 time slot protocol, not only because it typically has a high spectral efficiency [2], but also because novel differential modulation strategies can be derived.

Source terminal $i$ wants to transmit $s_{i}[n] \in \mathcal{Q}$ in time slot $n$, where $\mathcal{Q}$ is the constellation set. We assume that $M$-PSK is used, i.e., $\mathcal{Q}=\left\{e^{\jmath \theta} \mid \theta=\frac{2 \pi}{M} l, l=0,1, \ldots, M-1\right\}$. Suppose that 
the transmitted signals by the source $i$ and the relay at time slot $n$ are $x_{i}[n], i=1,2$ and $x_{r}[n]$, respectively. If conventional differential modulation is used, we have $x_{i}[n]=x_{i}[n-1] s_{i}[n]$. The received signal at the relay is

$$
y_{r}[n]=h_{1} x_{1}[n]+h_{2} x_{2}[n]+w_{r}[n],
$$

and the receive signal at terminal $i$ is

$$
y_{i}[n]=h_{i} x_{r}[n]+w_{i}[n],
$$

where $h_{i}$ is the channel gain between source $i$ and the relay $i=1,2, w_{r}[n]$ and $w_{i}[n]$ are additive white Gaussian noise (AWGN) at the relay and the source $i$ with zero mean and variances $\sigma_{r}^{2}$ and $\sigma_{s}^{2}$, respectively. We have assumed reciprocal channel for notation simplicity. Otherwise mentioned, we assume that $h_{i}$ remains static at least over two time slots.

By differential modulation, we do not assume the knowledge of $h_{1}$ and $h_{2}$ at any node. However, we assume that the statistics of $h_{i}$ is known, which is $\mathcal{C N}(0,1)$.

\section{B. Amplify-and-Forward}

By AF, we choose $x_{r}[n]=\beta y_{r}[n]$ where $\beta>0$ is a constant to keep the power constraint at the relay. We focus on source 1 as source 2 can be discussed similarly. At source 1, we obtain

$$
y_{1}[n]=\beta h_{1} h_{2} x_{2}[n]+\beta h_{1}^{2} x_{1}[n]+z_{1}[n],
$$

where $z_{1}[n]$ is AWGN with zero mean and variance $\sigma_{z}^{2}=$ $\beta^{2}\left|h_{1}\right|^{2} \sigma_{r}^{2}+\sigma_{s}^{2}$. When $h_{1}^{2}$ is known at source 1 , we can subtract the contribution of $x_{1}[n]$ as source 1 knows the signal it has sent. In this case, conventional differential modulation follows naturally as after cancelling $x_{1}[n]$ (3) actually becomes one way communication. The problem becomes complicated when $h_{1}^{2}$ is unknown at source 1 since it cannot cancel $\beta h_{1}^{2} x_{1}[n]$ without knowing $h_{1}^{2}$. Due to the mixture of the two signals, the conventional differential detection cannot be used.

1) Optimal ML Detector: The optimal ML detector should be derived from the pdf of $\mathbf{y}_{1}$ conditioned on $\mathbf{x}_{1}, \mathbf{x}_{2}$, where $\mathbf{y}_{1}=\left[y_{1}[n], y_{1}[n-1]\right]^{T}$ and $\mathbf{x}_{i}=\left[x_{i}[n-1], x_{i}[n]\right]^{T}, i=$ 1,2. Clearly, $\mathbf{y}_{1}$ is complex Gaussian given $h_{1}$ and $\mathbf{x}_{1}, \mathbf{x}_{2}$ with pdf $p\left(\mathbf{y}_{1} \mid h_{1}, \mathbf{x}_{1}, \mathbf{x}_{2}\right)$. We can obtain $p\left(\mathbf{y}_{1} \mid \mathbf{x}_{1}, \mathbf{x}_{2}\right)$ by averaging $p\left(\mathbf{y}_{1} \mid h_{1}, \mathbf{x}_{1}, \mathbf{x}_{2}\right)$ over $h_{1}$. The optimal ML detector is obtained by maximizing $p\left(\mathbf{y}_{1} \mid \mathbf{x}_{1}, \mathbf{x}_{2}\right)$. However, closed-form $p\left(\mathbf{y}_{1} \mid \mathbf{x}_{1}, \mathbf{x}_{2}\right)$ is hard to obtain. Details can be found in [8].

2) Suboptimal ML Detector: In the suboptimal detector, we first cancel the contribution of $x_{1}[n]$ in $y_{1}[n]$. We can subtract the contribution of $x_{1}[n]$ in $y_{1}[n]$ via

$$
\begin{aligned}
u_{1}[n] & =y_{1}[n]-y_{1}[n-1] s_{1}[n] \\
& =\beta h_{1} h_{2} x_{2}[n-1]\left(s_{2}[n]-s_{1}[n]\right)+z_{1}[n]-s_{1}[n] z_{1}[n-1] .
\end{aligned}
$$

Given $h_{1}$ and $s_{2}[n]-s_{1}[n], u_{1}[n]$ is complex Gaussian with pdf $p\left(u_{1}[n] \mid h_{1}, s_{2}[n], s_{1}[n]\right)$. To obtain the pdf of $u_{1}[n]$ conditional only on $s_{2}[n]-s_{1}[n]$, we need to integrate $p\left(u_{1}[n] \mid h_{1}, s_{2}[n], s_{1}[n]\right)$ over the pdf of $h_{1}$, i.e., [8]

$$
\begin{aligned}
& p\left(u_{1}[n] \mid s_{2}[n], s_{1}[n]\right)=\int p\left(u_{1}[n] \mid h_{1}, s_{2}[n], s_{1}[n]\right) p\left(h_{1}\right) d h_{1} \\
&= \frac{1}{\pi \beta^{2}\left(\left|s_{2}[n]-s_{1}[n]\right|^{2}+2 \sigma_{r}^{2}\right)} \exp \left(\frac{2 \sigma_{s}^{2}}{\beta^{2}\left(\left|s_{2}[n]-s_{1}[n]\right|^{2}+2 \sigma_{r}^{2}\right)}\right) \\
& \times K_{0}\left(2 \sqrt{\frac{\left|u_{1}[n]\right|^{2}}{\beta^{2}\left(\left|s_{2}[n]-s_{1}[n]\right|^{2}+2 \sigma_{r}^{2}\right)}}\right),
\end{aligned}
$$

where $K_{0}(\cdot)$ is the modified bessel function of the second kind of zero order. Therefore, the suboptimal ML detector is

$$
\hat{s}_{2}[n]=\underset{s_{2}[n]}{\operatorname{argmax}} p\left(u_{1}[n] \mid s_{2}[n], s_{1}[n]\right) .
$$

The suboptimal detector requires that $\forall s_{1}[n] \in \mathcal{Q} \mid s_{2}[n]-$ $\left.s_{1}[n]\right|^{2} \neq\left|s_{2}^{\prime}[n]-s_{1}[n]\right|^{2}, \forall s_{2}[n], s_{2}^{\prime}[n] \in \mathcal{Q}$ and $s_{2}[n] \neq s_{2}^{\prime}[n]$. Clearly, BPSK satisfies this condition. For general $M$-PSK, we could rotate the constellation to satisfy this condition. By deriving the joint pdf of $u_{1}[n], \ldots, u_{1}[n-K]$, we can obtain a multiple symbol detector.

3) Decision Feedback Detector: By assuming perfect detection in previous time slots, we get

$$
\begin{aligned}
d[n]= & x_{2}[n-2]\left(s_{2}[n-1]-s_{1}[n-1]\right) u_{1}[n] \\
& -x_{2}[n-1]\left(s_{2}[n]-s_{1}[n]\right) u_{1}[n-1] \\
= & x_{2}[n-2]\left(s_{2}[n-1]-s_{1}[n-1]\right)\left(z_{1}[n]-s_{1}[n] z_{1}[n-1]\right) \\
& -x_{2}[n-1]\left(s_{2}[n]-s_{1}[n]\right)\left(z_{1}[n-1]-s_{1}[n-1] z_{1}[n-2]\right) .
\end{aligned}
$$

We can thus obtain the pdf of $d[n]$ as

$$
p\left(d[n] \mid s_{1}[n-1], s_{1}[n], s_{2}[n-1], s_{2}[n]\right)=\frac{1}{\pi \hat{\sigma}^{2}} e^{-\frac{|d[n]|^{2}}{\hat{\sigma}^{2}}},
$$

where

$$
\begin{aligned}
\hat{\sigma}^{2}=\left(\left|s_{2}[n-1]-s_{1}[n-1]\right|^{2}+\left|s_{2}[n]-s_{1}[n]\right|^{2}\right) \sigma_{z}^{2} \\
+\left|s_{2}[n] s_{2}[n-1]-s_{1}[n] s_{1}[n-1]\right|^{2} \sigma_{z}^{2} .
\end{aligned}
$$

The decision feedback detector is obtained by maximizing (8). As $\sigma_{z}^{2}$ depends on $\left|h_{1}\right|^{2}$, one way to remove such dependence is to integrate (8) over $h_{1}$. Another way is simply replacing $\left|h_{1}\right|^{2}$ with $E\left\{\left|h_{1}\right|^{2}\right\}=1$. Note that $s_{2}[n]$ also appears in $\hat{\sigma}^{2}$. To reduce the complexity, a suboptimal detector is to minimize $|d[n]|^{2}$ only. This detector requires that $s_{2}[n]-$ $s_{1}[n] \neq 0, \forall s_{2}[n], s_{1}[n] \in \mathcal{Q}$. Hence, the two source terminals should transmit with different constellations. For example, we could choose $\mathcal{Q}_{1}=\{-1,1\}$ and $\mathcal{Q}_{2}=\{-\jmath, \jmath\}$ for the two source terminals. When $M$-PSK is used, the constellation for one terminal is a rotation of that for the other one.

4) Prediction Based Detector: When channel gains $h_{1}$ and $h_{2}$ varies over time, the contribution of $x_{1}[n]$ cannot be completely cancelled out by using (4) directly. Also, it is hard to compute $p\left(\mathbf{y}_{1} \mid \mathbf{x}_{1}, \mathbf{x}_{2}\right)$ in time varying channels. Motivated by the prediction based decision feedback differential detection in [5] for the point to point case, we propose a prediction based differential detector for TWRC. Instead of subtracting $y_{1}[n-1] s_{1}[n]$ from $y_{1}[n]$, we consider cancelling the effect of $x_{1}[n]$ by using $K$ previously received symbols, i.e.,

$$
u_{1}[n]=y_{1}[n]-\sum_{k=1}^{K} p_{n, k} y_{1}[n-k] \prod_{i=0}^{k-1} s_{1}[n-i] .
$$

We should choose $p_{n, k}$ to minimize the expected noise variance and estimation error $w_{e}=u_{1}[n]-\beta h_{1} h_{2} x_{2}[n]$. Hence, $p_{n, k}$ can be determined from the Yule-Walker equations

$$
\mathbf{p}_{n}=\left(\check{\mathbf{C}}_{h 1}+\left(\sigma_{r}^{2}+\frac{\sigma_{s}^{2}}{\beta^{2}}\right) \mathbf{I}_{K}\right)^{-1} \mathbf{b},
$$

where $\quad \check{\mathbf{C}}_{h 1}=\left[E\left\{h_{1}^{2}[n-i]\left(h_{1}^{2}[n-j]\right)^{*}\right\}\right] \quad$ and $\quad \mathbf{b}=$ $\left[E\left\{h_{1}^{2}[n]\left(h_{1}^{2}[n-1]\right)^{*}\right\}, \quad \cdots, E\left\{h_{1}^{2}[n]\left(h_{1}^{2}[n-K]\right)^{*}\right\}\right]^{T}$. With this $\mathbf{p}_{n}$, we can write $u_{1}[n]$ in (10) as 


$$
\begin{aligned}
& u_{1}[n]=\beta h_{1}[n] h_{2}[n] x_{2}[n] \\
& \quad-\beta \sum_{k=1}^{K} p_{n, k} h_{1}[n-k] h_{2}[n-k] x_{2}[n-k] \prod_{i=0}^{k-1} s_{1}[n-i]+w_{e} .
\end{aligned}
$$

The joint distribution of $h_{1}[n] h_{2}[n], \ldots, h_{1}[n-K] h_{2}[n-K]$ is unknown. We approximate $u_{1}[n]$ as a complex Gaussian random variable with zero mean and variance

$$
\begin{aligned}
\sigma_{u 1}^{2}\left(s_{2}[n], \ldots, s_{2}[n-K]\right) & =\beta^{2} \check{\mathbf{s}}^{H} \check{\mathbf{C}}_{h} \check{\mathbf{s}}+\beta^{2}\left(2+\sigma_{r}^{2}\right)+\sigma_{s}^{2} \\
& -\mathbf{b}^{H}\left(\check{\mathbf{C}}_{h 1}+\left(\sigma_{r}^{2}+\frac{\sigma_{s}^{2}}{\beta^{2}}\right) \mathbf{I}_{K}\right)^{-1} \mathbf{b},
\end{aligned}
$$

where $\mathbf{s}=\left[1, s_{1}[n] s_{2}^{*}[n], \ldots, \prod_{i=0}^{K-1} s_{1}[n-i] s_{2}^{*}[n-i]\right]^{T}$ and $\check{\mathbf{C}}_{h}=$ $\left[E\left\{h_{1}[n-i] h_{1}^{*}[n-j]\right\} E\left\{h_{2}[n-i] h_{2}^{*}[n-j]\right\}\right]$. By using decision feedback, we assume that $s_{2}[n-1] \ldots, s_{2}[n-K]$ have been detected correctly, which are denoted as $\hat{s}_{2}[n-1] \ldots, \hat{s}_{2}[n-$ $K]$. The detector for $s_{2}[n]$ can be obtained by maximizing $p\left(u_{1}[n] \mid s_{2}[n], \hat{s}_{2}[n-1], \ldots, \hat{s}_{2}[n-K]\right)$. The prediction based detector can also be extended to multiple symbol detection.

\section{Decode-and-Forward}

1) Single Symbol Detector: In DF, the relay first decodes its received signal. From (1), we can write the received signals at the relay in the $n$-th and $n$-1-th time slots as

$$
\mathbf{y}_{r}=h_{1} \mathbf{X}_{1} \mathbf{1}+h_{2} \mathbf{X}_{2} \mathbf{1}+\mathbf{w}_{r},
$$

where $\mathbf{y}_{r}=\left[y_{r}[n-1], y_{r}[n]\right]^{T}, \mathbf{X}_{i}=\operatorname{diag}\left\{x_{i}[n-1], x_{i}[n]\right\}, i=$ $1,2, \mathbf{1}=[1,1]^{T}$ and $\mathbf{w}_{r}=\left[w_{r}[n-1], w_{r}[n]\right]^{T}$. Therefore, $\mathbf{y}_{1}$ is complex Gaussian given $x_{1}, x_{2}$, i.e.,

$$
p\left(\mathbf{y}_{r} \mid \mathbf{x}_{1}, \mathbf{x}_{2}\right)=\frac{1}{(2 \pi)^{2} \operatorname{det}(\mathbf{C})} \exp \left(-\mathbf{y}_{r}^{H} \mathbf{C}^{-1} \mathbf{y}_{r}\right)
$$

where

$$
\mathbf{C}=\left[\begin{array}{cc}
2 & s_{1}^{*}[n]+s_{2}^{*}[n] \\
s_{1}[n]+s_{2}[n] & 2
\end{array}\right]+\sigma_{r}^{2} \mathbf{I}_{2} .
$$

Let $s[n]=s_{1}[n]+s_{2}[n]$. We can write (15) as

$$
\begin{array}{r}
p\left(\mathbf{y}_{r} \mid s[n]\right)=\frac{1}{(2 \pi)^{2}\left(\left(2+\sigma_{r}^{2}\right)^{2}-|s[n]|^{2}\right)} \exp \left(-\frac{\left(1+\sigma_{r}^{2}\right)\left|y_{r}[n]\right|^{2}}{\left(2+\sigma_{r}^{2}\right)^{2}-|s[n]|^{2}}\right. \\
\left.-\frac{\left(2+\sigma_{r}^{2}-\left|s[n]^{2}\right|\right)\left|y_{r}[n-1]\right|^{2}+\left|y_{r}[n]-y_{r}[n-1] s[n]\right|^{2}}{\left(2+\sigma_{r}^{2}\right)^{2}-|s[n]|^{2}}\right) .
\end{array}
$$

As several $s_{1}[n]$ and $s_{2}[n]$ pairs may give the same $s_{1}[n]+$ $s_{2}[n]$, we should use the maximum a posteriori (MAP) detector by maximizing $p\left(s[n] \mid \mathbf{y}_{r}\right)=\frac{p\left(\mathbf{y}_{r} \mid s[n]\right) p(s[n])}{p\left(\mathbf{y}_{r}\right)}$. As $p\left(\mathbf{y}_{r}\right)$ is a common factor, the MAP detector for $s[n]$ is

$$
\hat{s}[n]=\underset{s[n]=s_{1}[n]+s_{2}[n], s_{1}[n], s_{2}[n] \in \mathcal{Q}}{\operatorname{argmax}} p\left(\mathbf{y}_{r} \mid s[n]\right) p(s[n]) .
$$

After obtaining $\hat{s}[n]=\hat{s}_{1}[n]+\hat{s}_{2}[n]$ from (18), the relay does not transmit a scaled version of $\hat{s}[n]$ as in conventional DF because we should also use differential modulation in the downlink channel from the relay to the two source terminals.

We use the idea of network coding. Recall that in the basic network coding, source terminal $i$ transmits $b_{i} \in\{0,1\}$. The relay decodes $b_{1}$ and $b_{2}$ and broadcasts $b_{r}=\left(b_{1}+b_{2}\right) \bmod 2$. As source $i$ already knows $b_{i}$, it can decode the signal from the other terminal by $\left(b_{r}-b_{i}\right) \bmod 2$. In conventional network coding, the relay decodes $b_{1}$ and $b_{2}$ separately at the physical layer and network coding is applied at the networking layer.

To use network coding at the physical layer, we first solve $\hat{s}[n]=\hat{s}_{1}[n]+\hat{s}_{2}[n]$ from (18) by exhausting $s_{1}[n]=e^{J \frac{2 \pi}{M} l_{1}}$ and $s_{2}[n]=e^{\jmath \frac{2 \pi}{M} l_{2}}, l_{i} \in\{0,1, \ldots, M-1\}$ and $i=1,2$. A mapping $\mathcal{M}$ is then used to map $\hat{s}[n]$ to $s_{r}[n]=\mathcal{M}(\hat{s}[n])$. By differential modulation, the relay then broadcasts $x_{r}[n]=x_{r}[n-1] s_{r}[n]$. As source terminal $i$ knows $s_{i}[n]$, it can decode signals from the other terminal if it receives $s_{1}[n]+s_{2}[n]$. Therefore, the mapping should satisfy the condition $\mathcal{M}\left(s_{1}[n]+\right.$ $\left.s_{2}[n]\right) \neq \mathcal{M}\left(s_{1}[n]+s_{2}^{\prime}[n]\right)$ and $\mathcal{M}\left(s_{1}[n]+s_{2}[n]\right) \neq \mathcal{M}\left(s_{1}^{\prime}[n]+\right.$ $\left.s_{2}[n]\right), \forall s_{1}[n] \neq s_{1}^{\prime}[n], s_{2}[n] \neq s_{2}^{\prime}[n]$. To find the mapping $\mathcal{M}$, we need the following lemma proved in [8].

Lemma 1: For any $p_{1}, p_{2}, q_{1}, q_{2} \in\{0,1, \ldots, M-1\}, e^{\jmath \frac{2 \pi}{M} p_{1}}+$ $e^{\jmath \frac{2 \pi}{M} p_{2}}=e^{\jmath \frac{2 \pi}{M} q_{1}}+e^{\jmath \frac{2 \pi}{M} q_{2}}$ if $\left(p_{1}-p_{2}\right) \bmod M=\frac{M}{2}$ and $\left(q_{1}-\right.$ $\left.q_{2}\right) \bmod M=\frac{M}{2}$ or $p_{1} \bmod M=q_{1} \bmod M$ and $p_{2} \bmod M=q_{2}$ $\bmod M$ or $p_{1} \bmod M=q_{2} \bmod M$ and $p_{1} \bmod M=q_{2} \bmod M$.

To find the mapping, we construct a graph $\mathcal{G}$ as in [3], where each node corresponds to $s_{1}[n]+s_{2}[n]$ and there is an edge between $s_{1}[n]+s_{2}[n]$ and $s_{1}[n]+s_{2}^{\prime}[n]$ and an edge between $s_{1}[n]+s_{2}[n]$ and $s_{1}^{\prime}[n]+s_{2}[n], \forall s_{1}[n] \neq s_{1}^{\prime}[n], s_{2}[n] \neq$ $s_{2}^{\prime}[n]$. Then, the mapping $\mathcal{M}$ corresponds to a coloring of graph $\mathcal{G}$. We assume that $M$ colors are used. When $M$ is odd, there do not exist $l_{1}$ and $l_{2}$ such that $\left(l_{1}-l_{2}\right) \bmod M=$ $\frac{M}{2}$. From Lemma 1 , each node in $\mathcal{G}$ only corresponds to $s_{1}[n]+s_{2}[n]$ and $s_{2}[n]+s_{1}[n]$. Therefore, the degree of each node is $2 M-1$ and each node lies exactly in two cliques, where each clique contains $M$ nodes. We show that the graph can be colored greedily using $M$ colors. We first pick an arbitrary node and assign a color to this node. Then, the remaining $M-1$ colors are assigned to $M-1$ nodes in the two cliques, respectively. Note that all the nodes in the first clique corresponds to $s_{1}[n]+s_{2}^{\prime}[n]$ while they corresponds to $s_{1}^{\prime}[n]+s_{2}[n]$ in the second clique. Therefore, there is not edge between nodes in the first clique and nodes in the second clique as $s_{1}[n] \neq s_{1}^{\prime}[n], s_{2}[n] \neq s_{2}^{\prime}[n]$. The greedy coloring does not void the coloring condition. We then apply the same process to each node. It is easy to see that we can obtain a valid coloring with $M$ colors in the end. When $M$ is even, all $s_{1}[n]$ and $s_{2}[n]$ such that $\left(l_{1}-l_{2}\right) \bmod M=\frac{M}{2}$ corresponds to the same node, i.e., $s_{1}[n]+s_{2}[n]$. We color this node using a color out of the $M$ colors and remove this node from $\mathcal{G}$, which gives a graph $\mathcal{G}^{\prime}$. In $\mathcal{G}^{\prime}$, each node belongs to two cliques of size $M-1$. By following the same greedy coloring algorithm as when $M$ is odd, $\mathcal{G}^{\prime}$ can be colored using $M-1$ colors. Finally, $M$ colors are sufficiently to color $\mathcal{G}$. Therefore, we can obtain a valid mapping $\mathcal{M}$ for any $M$. For example, when $\mathcal{Q}=\{-1,1\}$,

$$
\mathcal{M}(\hat{s}[n])=\left\{\begin{array}{cc}
1, & \text { if } \hat{s}[n]=0, \\
-1, & \text { otherwise, }
\end{array}\right.
$$

Different from conventional network coding at the networking layer operating on finite field. Here, we define a physical layer differential network coding by a mapping $\mathcal{M}$. But $\mathcal{M}$ is operated on real signals rather than on finite filed. In fact, the mapping $\mathcal{M}$ defines a group with an equivalent additive operation on the indices as

$$
l_{r}=l_{1} \oplus l_{2} .
$$

The signal transmitted by the relay is thus $s_{r}[n]=e^{\jmath \frac{2 \pi}{M}} l_{r}$. The operation (20) is called differential network coding at the physical layer. 
In the downlink, the received signal at source 1 is

$$
y_{1}[n]=h_{1} x_{r}[n]+w_{1}[n]=y_{1}[n-1] s_{r}[n]+z_{1}[n],
$$

where $z_{1}[n]=w_{1}[n]-w_{1}[n-1] \hat{s}_{1}[n] \hat{s}_{2}^{*}[n]$ is complex Gaussian with zero mean and variance $2 \sigma_{s}^{2}$. Let $P_{e}\left(\left\{s_{1}[n], s_{2}[n]\right\} \rightarrow\right.$ $\left.\left\{\tilde{s}_{1}[n], \tilde{s}_{2}[n]\right\}\right)$ be the pairwise error probability (PEP) that $s_{1}[n]=e^{\jmath \frac{2 \pi}{M} l_{1}}$ and $s_{2}[n]=e^{j \frac{2 \pi}{M} l_{2}}$ are transmitted but $\tilde{s}_{1}[n]=$ $e^{\jmath \frac{2 \pi}{M} l_{1}}$ and $\tilde{s}_{2}[n]=e^{\jmath \frac{2 \pi}{M} \tilde{l}_{2}}$ are decoded by the relay and $l_{1} \oplus l_{2} \neq$ $\tilde{l}_{1} \oplus \hat{l}_{2}$. The probability that $y_{1}[n]$ and $y_{1}[n-1]$ are received conditioned on $s_{1}[n]$ and $s_{2}[n]$ is thus

$$
\begin{aligned}
& \operatorname{Pr}\left(y_{1}[n], y_{1}[n-1] \mid s_{1}[n], s_{2}[n]\right) \\
= & \sum_{\tilde{s}_{1}[n], \tilde{s}_{2}[n]} P_{e}\left(\left\{s_{1}[n], s_{2}[n]\right\} \rightarrow\left\{\tilde{s}_{1}[n], \tilde{s}_{2}[n]\right\}\right) \operatorname{Pr}\left(y_{1}[n] \mid y_{1}[n-1], \tilde{s}_{r}[n]\right),
\end{aligned}
$$

where $\tilde{s}_{r}[n]=e^{j \frac{2 \pi}{M} \tilde{l}_{1} \oplus \hat{l}_{2}}$. By using the ML detector (17) at the relay, the PEP can be found in [8].

The ML detector for $s_{2}[n]$ can thus be obtained by maximizing $\operatorname{Pr}\left(y_{1}[n], y_{1}[n-1] \mid s_{1}[n], s_{2}[n]\right)$ given $s_{1}[n]$. It may be complicated to implement the true ML detector directly. In high signal-to-noise ratio (SNR), (22) is dominated by the term corresponding to that $s_{1}[n]+s_{2}[n]$ is decoded correctly. The suboptimal ML detector for $s_{r}[n]$ can be obtained as [7]

$$
\hat{s}_{r}[n]=\underset{s_{r}[n] \in \mathcal{Q}}{\operatorname{argmin}}\left|y_{1}[n]-y_{1}[n-1] s_{r}[n]\right|^{2} .
$$

Let $\hat{s}_{r}[n]=e^{\jmath \frac{2 \pi}{M} \hat{l}_{r}}$. We can then decode $l_{2}$ to be $\hat{l}_{2}=l_{r} \ominus l_{1}$, where $\ominus$ follows naturally from (20). The ML detector at source 2 can be obtained similarly.

2) Multiple Symbol Detector: Channel gains are now assumed to vary over time. We consider joint detection of $K$ symbols. Let $\mathbf{y}_{r}=\left[y_{r}[n], \ldots, y_{r}[n-K]\right]^{T}, \mathbf{h}_{i}=\left[h_{i}[n], \ldots, h_{i}[n-\right.$ $K]]^{T}, \mathbf{x}_{i}=\left[x_{i}[n], \ldots, x_{i}[n-K]\right]^{T}, \mathbf{X}_{i}=\operatorname{diag}\left\{\mathbf{x}_{i}\right\}, i=1,2$, and $\mathbf{w}_{r}=\left[w_{r}[n], \ldots, w_{r}[n-K]\right]^{T}$. We then have

$$
\mathbf{y}_{r}=\mathbf{X}_{1} \mathbf{h}_{1}+\mathbf{X}_{2} \mathbf{h}_{2}+\mathbf{w}_{r} .
$$

it is easy to see that $\mathbf{y}_{r}$ is a Gaussian vector with pdf

$$
p\left(\mathbf{y}_{r} \mid \mathbf{x}_{1}, \mathbf{x}_{2}\right)=\frac{1}{(2 \pi)^{K+1} \operatorname{det} \mathbf{C}} \exp \left(-\mathbf{y}_{r}^{H} \mathbf{C}^{-1} \mathbf{y}_{r}\right),
$$

where $\mathbf{C}=\mathbf{X}_{1} \mathbf{C}_{h 1} \mathbf{X}_{1}^{H}+\mathbf{X}_{2} \mathbf{C}_{h 2} \mathbf{X}_{2}^{H}+\sigma_{s}^{2} \mathbf{I}_{K+1}$

$$
=\mathbf{X}_{1}\left(\mathbf{C}_{h 1}+\mathbf{X}_{1}^{H} \mathbf{X}_{2} \mathbf{C}_{h 2} \mathbf{X}_{2}^{H} \mathbf{X}_{1}+\sigma_{s}^{2} \mathbf{I}_{K+1}\right) \mathbf{X}_{1}^{H},
$$

$\mathbf{C}_{h 1}=E\left\{\mathbf{h}_{1} \mathbf{h}_{1}^{H}\right\}$ and $\mathbf{C}_{h 2}=E\left\{\mathbf{h}_{2} \mathbf{h}_{2}^{H}\right\}$. Let $\mathbf{x}_{3}=\left[x_{1}[n] x_{2}^{*}[n]\right.$, $\left.\ldots, x_{1}[n-K] x_{2}^{*}[n-K]\right]^{T}$ and $\mathbf{X}_{3}=\mathbf{X}_{2}^{H} \mathbf{X}_{1}$. We can write $(25)$

$$
\begin{aligned}
& p\left(\mathbf{y}_{r} \mid \mathbf{x}_{1}, \mathbf{x}_{3}\right)=\frac{1}{(2 \pi)^{K+1} \operatorname{det}\left(\mathbf{C}_{h 1}+\mathbf{X}_{3}^{H} \mathbf{C}_{h 2} \mathbf{X}_{3}+\sigma_{s}^{2} \mathbf{I}_{K+1}\right)} \\
& \times \exp \left(-\mathbf{x}_{1}^{T} \mathbf{Y}_{r}^{H}\left(\mathbf{C}_{h 1}+\mathbf{X}_{3}^{H} \mathbf{C}_{h 2} \mathbf{X}_{3}+\sigma_{s}^{2} \mathbf{I}_{K+1}\right)^{-1} \mathbf{Y}_{r} \mathbf{x}_{1}^{*}\right),
\end{aligned}
$$

where $\mathbf{Y}_{r}=\operatorname{diag}\left\{\mathbf{y}_{r}\right\}$. By maximizing (27), we obtain the ML detector for $\mathbf{x}_{1}$ and $\mathbf{x}_{3}$. One remarkable property of (27) is that the detection for $\mathbf{x}_{1}$ and $\mathbf{x}_{3}$ is separable. For a given $\mathbf{x}_{3}$, we can solve $\mathbf{x}_{1}$ by

$$
\hat{\mathbf{x}}_{1}=\underset{\mathbf{x}_{1} \in \mathcal{Q}^{K+1}}{\operatorname{argmin}} \mathbf{x}_{1}^{T} \mathbf{Y}_{r}^{H}\left(\mathbf{C}_{h 1}+\mathbf{X}_{3}^{H} \mathbf{C}_{h 2} \mathbf{X}_{3}+\sigma_{s}^{2} \mathbf{I}_{K+1}\right)^{-1} \mathbf{Y}_{r} \mathbf{x}_{1}^{*} .
$$

Note that (28) lies in the so-called integer least squares programming and can be solved efficiently using the sphere decoder (SD) for $M$-PSK [9] and semidefinite programming (SDP) for $M$-PSK [10]. Even though SD or SDP can be used to solve (28) efficiently, we need to solve (28) for every possible $\mathbf{x}_{3}$, which may be infeasible when $K$ is large. To save complexity, we decouple the detection of $\mathbf{x}_{1}$ and $\mathbf{x}_{3}$. We first solve a relaxed problem of (28) by replacing the finite constellation constraint with $\mathbf{x}_{1}^{H} \mathbf{x}_{1}=$ $K+1$, whose minimum value can be easily obtained by Rayleigh quotient theory as $(K+1) \lambda_{\min }(\mathbf{A})$, where $\mathbf{A}=$ $\mathbf{Y}_{r}^{H}\left(\mathbf{C}_{h 1}+\mathbf{X}_{3}^{H} \mathbf{C}_{h 2} \mathbf{X}_{3}+\sigma_{s}^{2} \mathbf{I}_{K+1}\right)^{-1} \mathbf{Y}_{r}$ and $\lambda_{\min }(\mathbf{A})$ is the minimum eigenvalue of $\mathbf{A}$. Then $\mathbf{x}_{3}$ can be obtained by

$$
\hat{\mathbf{x}}_{3}=\underset{\mathbf{x}_{3} \in \mathcal{Q}^{K+1}}{\operatorname{argmin}} \frac{\exp \left(-(K+1) \lambda_{\min }(\mathbf{A})\right)}{(2 \pi)^{K+1} \operatorname{det}\left(\mathbf{C}_{h 1}+\mathbf{X}_{3}^{H} \mathbf{C}_{h 2} \mathbf{X}_{3}+\sigma_{s}^{2} \mathbf{I}_{K+1}\right)} .
$$

After solving (29), we substitute $\hat{\mathbf{x}}_{3}$ into (28) to obtain $\hat{\mathbf{x}}_{1}$. The transmitted signal is thus $\mathbf{s}_{r}=\mathcal{M}\left(\left(\mathbf{I}_{K+1}+\operatorname{diag}\left\{\hat{\mathbf{x}}_{3}\right\}\right) \hat{\mathbf{x}}_{1}\right)$, where $\mathcal{M}$ is defined in (19).

By assuming that $s_{i}[n], \ldots, s_{i}[n-K], i-1,2$, are decoded correctly at the relay for simplicity without considering (22), the multiple symbol differential detection for $\mathbf{s}_{r}$ at the source terminals can be obtained as in [5], [7]. Finally, source 1 recovers the signal from the other terminal via $\hat{l}_{2}=l_{r} \ominus l_{1}$.

\section{Remarks:}

- We have assume that the channel gains have the identical distribution. Both proposed AF and DF strategies can be readily extended to the case when the channel gains have different variances. In particular, in DF, the relay can still use a mapping $\mathcal{M}\left(\hat{s}_{1}[n], \hat{s}_{2}[n]\right)$ with slight modification of the mapping (19) by using the same way as in [3].

- Traditional network coding [11] is performed on finite field. In this paper, network coding extends naturally from operating on finite field in networking layer to operating on finite group in physical layer in DF protocols. Hence, the DF differential protocols can be considered to be differential network coding on physical layer.

\section{Multiple Antenna Systems}

In this section, we briefly discuss DUSTM when each node has multiple antennas. We assume that both sources have $N$ antennas and the relay has $M$ antennas. The input-output relationship at the relay at time slot $n$ can be written as

$$
\mathbf{Y}_{r}[n]=\mathbf{X}_{1}[n] \mathbf{H}_{1}[n]+\mathbf{X}_{2}[n] \mathbf{H}_{2}[n]+\mathbf{W}_{r}[n],
$$

where $\mathbf{X}_{i}[n]$ is the transmitted $T \times N$ matrix by source $i$ during time slot $n, \mathbf{Y}_{r}[n]$ is the received $T \times M$ matrix at the relay, $\mathbf{H}_{i}[n]$ is the $N \times M$ channel matrix between source $i$ and the relay, and $\mathbf{W}_{r}[n]$ is the $T \times N$ noise matrix, $i=1,2$. Similarly, the received signal at source $i$ at time slot $n$ is

$$
\mathbf{Y}_{i}[n]=\mathbf{X}_{r}[n] \mathbf{H}_{i}^{T}[n]+\mathbf{W}_{i}[n] .
$$

The definition of these matrices are similar to those in (30). By using $\mathrm{AF}$, the relay simply transmits $\mathbf{X}_{r}[n]=\beta \mathbf{Y}_{r}[n]$, where $\beta$ is a scalar to keep the average power constraint at the relay. All the strategies in Section II-B can be extended. By DF, the relay tries to decode its received signal. The strategies are similar to those in Section II-C. Note that in both AF and DF the ML detector can be solved by using variants of BID [7]. 
By maximizing the worst case pairwise error probability, the modulation design criterion for two-way DUSTM is to maximize the modified diversity product [8]

$$
\xi=\min _{\substack{0 \leq l_{1}<l_{1}^{\prime} \leq L-1,1 \\ 0 \leq l_{2}<l_{2}^{\prime} \leq L-1, l_{1} \oplus l_{2} \neq l_{1}^{\prime} \oplus l_{2}^{\prime}}} \prod_{i=1}^{2 N} \mu_{i}^{-\frac{1}{2}}\left(1+\mu_{i}\right)^{M}
$$

where $\mu_{i}$ is the singular value of

$$
\begin{aligned}
& \text { where } \mu_{i} \text { is the singular value of } \\
& {\left[\begin{array}{cc}
\left(4 \mathbf{I}_{N}-\mathbf{S}^{H} \mathbf{S}^{\prime}\right)\left(4 \mathbf{I}_{N}-\left(\mathbf{S}^{\prime}\right)^{H} \mathbf{S}^{\prime}\right)^{-1} & 2\left(\mathbf{S}-\mathbf{S}^{\prime}\right)^{H}\left(4 \mathbf{I}_{N}-\mathbf{S}^{\prime}\left(\mathbf{S}^{\prime}\right)^{H}\right)^{-1} \\
2\left(\mathbf{S}-\mathbf{S}^{\prime}\right)\left(4 \mathbf{I}_{N}-\left(\mathbf{S}^{\prime}\right)^{H} \mathbf{S}^{\prime}\right)^{-1} & \left(4 \mathbf{I}_{N}-\mathbf{S}\left(\mathbf{S}^{\prime}\right)^{H}\right)\left(4 \mathbf{I}_{N}-\mathbf{S}^{\prime}\left(\mathbf{S}^{\prime}\right)^{H}\right)^{-1}
\end{array}\right]}
\end{aligned}
$$

and $\mathbf{S}[n]=\mathbf{S}_{l_{1}}+\mathbf{S}_{l_{2}}, \mathbf{S}^{\prime}[n]=\mathbf{S}_{l_{1}^{\prime}}+\mathbf{S}_{l_{2}^{\prime}}$. One simple choice of constellation is that the constellation of one source terminal is a phase rotation of that of the other one.

\section{Simulation Results}

In this section, we compare the performance of the proposed differential decoding strategies with those by partitioning each time slot into 4 parts and using conventional differential decoding, where the former is denoted as 2-AF (DF) and the latter is denoted as 4-AF (DF). The transmission power of each node is fixed to be 1 . In addition, we choose $\sigma_{r}^{2}=\sigma_{s}^{2}$. The SNR is defined as $1 / \sigma_{r}^{2}$. In the following, symbol error rate (SER) is used to compare different strategies. We only consider single antenna systems. More simulation results such as those for multiple antenna systems can be found in [8].

Fig. 1 compares SER of 2-AF strategies with 4-AF. The detector (6) is denoted as "Suboptimal ML". Decision feedback using (9) and using $|d[n]|^{2}$ only with perfectly decoded previous symbols are denoted as "Genie Decision Feedback" and "Genie Suboptimal Decision Feedback", respectively, while decision feedback using $|d[n]|^{2}$ only and decoded previous symbols is denoted as "Non-Genie Decision Feedback". In suboptimal ML, BPSK is used at both terminals, while $\{-1,1\}$ and $\{-\jmath, \jmath\}$ are used at each terminal in decision feedback detectors. We also include 2-AF with perfect $h_{i}^{2}$ such that the self interference signal can be cancelled and conventional differential decoding can be used. As 4-AF consumes 2 times more time than 2-AF, we compare 2-AF with 4-AF using QPSK, where both strategies have the same average transmission rate. Suboptimal ML performs worse than decision feedback detectors because 2 received signals are used in suboptimal ML while 3 received signals are used in decision feedback detectors. We also find that the decision feedback detectors perform close to 2-AF with perfect $h_{i}^{2}$ even though $h_{i}^{2}$ is unknown in the former case. With genie, we observe that compared with decision feedback using (9) the performance degradation by using $|d[n]|^{2}$ only is small, which suggests that using $|d[n]|^{2}$ only is a good choice in practice due to its simplicity. Without genie, there is an additional 1-dB loss at $\mathrm{SER}=10^{-2}$ by using $|d[n]|^{2}$. At $\mathrm{SER}=10^{-2}$, non-genie decision feedback has a 0.5 -dB gain over $4-\mathrm{AF}$, which shows the advantage of 2 -AF protocols.

\section{Conclusion}

In this paper, we have considered non-coherent transmission in TWRC. Differential AF and DF strategies were proposed for

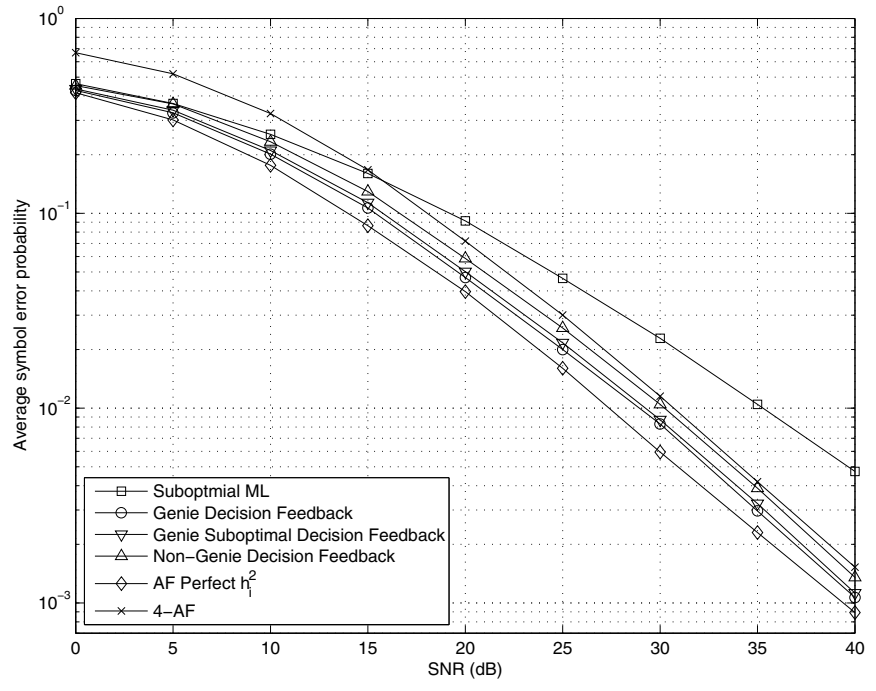

Fig. 1. Average symbol error rate comparison between different 2-AF strategies using BPSK and 4-AF protocol using QPSK.

both single antenna and multiple antenna systems. These new schemes are the counterpart of the traditional non-coherent detection or the differential detection in point to point communications. We derived ML detectors for both AF and DF protocols. The DF protocol could be considered as performing differential network coding at the physical layer. To reduce the complexity of the ML detector, decision feedback detectors and prediction based detectors were proposed. Moreover, the protocols were extended to the multiple-antenna case, where DUSTM design criterion is provided.

\section{REFERENCES}

[1] B. Rankov and A. Wittneben, "Spectral efficient signaling for halfduplex relay channels," in Proc. of Asilomar Conference on Signals, Systems and Computers, Oct. 2005, pp. 1066-1071.

[2] T. Cui, F. Gao, T. Ho, and A. Nallanathan, "Distributed space-time coding for two-way wireless relay networks," in Proc. of IEEE ICC, May 2008.

[3] T. Cui, T. Ho, and J. Kliewer, "Some results on relay strategies for memoryless two-way relay channels," in Proc. of Information Theory and Applications Workshop, Jan. 2008.

[4] _ "Relay strategies for memoryless two-way relay channels: Performance analysis and optimization,' in Proc. of IEEE ICC, May 2008.

[5] R. Schober, W. Gerstacker, and J. Huber, "Decision-feedback differential detection of MDPSK for flat Rayleighfading channels," IEEE Trans. Commun., vol. 47, no. 7, pp. 1025-1035, Jul. 1999.

[6] B. M. Hochwald and W. Sweldens, "Differential unitary space-time modulation," IEEE Trans. Commun., vol. 48, no. 12, pp. 2041-2052, Dec. 2000.

[7] T. Cui and C. Tellambura, "Bound intersection detection for multiplesymbol differential unitary space-time modulation," IEEE Trans. Commun., vol. 53, no. 12, pp. 2114 - 2123, Dec. 2005.

[8] T. Cui, F. Gao, and C. Tellambura, "Differential modulation for twoway wireless communications: Differential network coding at physical layer," Caltech, Tech. Rep., Mar. 2008.

[9] T. Cui and C. Tellambura, "Joint data detection and channel estimation for OFDM systems," IEEE Trans. Commun., vol. 54, no. 4, pp. 670-679, April 2006.

[10] W.-K. Ma, P.-C. Ching, and Z. Ding, "Semidefinite relaxation based multiuser detection for M-ary PSK multiuser systems," IEEE Trans. Signal Processing, vol. 52, no. 10, pp. 2862 - 2872, Oct. 2004

[11] R. Ahlswede, N. Cai, S. Y. R. Li, and R. W. Yeung, "Network information flow," IEEE Trans. Inform. Theory, vol. 46, no. 4, pp. 1204 1216, Jul. 2000 . 